\title{
1 VipA N-terminal linker and VipB-VipB interaction modulate the contraction of Type VI secretion
}

\section{2 system sheath.}

3 Maximilian Brackmann¹, Jing Wang ${ }^{1}$, and Marek Basler ${ }^{1 *}$

4 1. Focal Area Infection Biology, Biozentrum, University of Basel, Klingelbergstrasse 50/70, CH -

54056 Basel, Switzerland

* Correspondence to: marek.basler@unibas.ch

\section{Abstract}

9 Secretion systems are essential for bacteria to survive and manipulate their environment. The bacterial Type VI Secretion System (T6SS) generates the force needed for protein translocation by the contraction of a long polymer called sheath, which is composed of interconnected

12 VipA/VipB subunits forming a six-start helix. The mechanism of T6SS sheath contraction and the

13 structure of its extended state are unknown. Here we show that elongating the N-terminal VipA

14 linker or eliminating charge of a specific VipB residue abolished sheath contraction and delivery

15 of effectors into target cells. The assembly of the non-contractile sheaths was dependent on the

16 baseplate component TSSE and mass-spectrometry analysis identified Hcp, VgrG and other

17 components of the T6SS baseplate specifically associated with stable non-contractile sheaths.

18 The ability to lock T6SS in the pre-firing state opens new possibilities for understanding its mode 19 of action. 
22 Various protein nanomachines have evolved to translocate macromolecules across biological

23 membranes. A subset of these nanomachines is composed of a rigid tube surrounded by a

24 contractile sheath attached to a baseplate. The sheath is initially assembled in a high energy,

25 extended state and then quickly transits to a low energy, contracted state, which results in

26 physical puncturing of the target membrane associated with the baseplate. The bacterial Type VI

27 secretion system (T6SS) uses this mechanism to deliver proteins across membranes [1-5].

28 Assembly of all contractile nanomachines starts by formation of a baseplate, which initiates polymerization of the inner tube. The tube serves as a template for quick assembly of the extended sheath [6-10]. Unlike other contractile systems, the assembly of T6SS baseplate requires the interaction with a membrane complex, which anchors the system to the cell envelope $[11,12]$. Two different classes of TsSA molecules are important for initiation of T6SS sheath assembly and its elongation [13,14], which progresses across the whole cell and thus allows the use of live-cell fluorescence microscopy to monitor the sheath dynamics $[1,12,15-17]$.

The sheath can be described as a six-start helix or as a stack of rings composed of six subunits, which are interconnected by $\mathrm{N}$ - and C-terminal linkers in the inner layer of the sheath. Inner

37 layers of sheaths of contractile phage, R-type pyocin and T6SS likely maintain the connectivity of the sheath during contraction and are evolutionarily related to each other. However, the surface

39 exposed domains are distinct [9,18-23]. Specifically, T6SS sheath contains a specific surface 
41 The mechanism of T6SS sheath contraction is unknown because the sheath contracts during

42 isolation from cells and thus the extended sheath is yet to be analyzed in detail [1].

43 Here, we identified two structural features of the T6SS sheath that play a critical role in its

44 contraction. Using live-cell fluorescence microscopy, we show that a single negatively charged

45 residue located on the surface of the middle domain of the T6SS sheath is critical for sheath

46 contraction but not sheath assembly. We further show that VipA N-terminal linker structure is

47 critical for sheath contraction. Insertion of two and more amino acid residues into this linker

48 completely abrogated contraction and allowed us to isolate non-contractile sheaths from cells

49 for mass-spectrometry and electron microscopy analysis. This analysis revealed that non-

50 contracted sheaths are stably associated with Hcp tube and components of T6SS baseplate.

51 Overall, our analysis shows that conserved structural features are involved in sheath contraction

52 and provides insights into T6SS assembly and mode of action.

\section{$54 \quad$ Results}

\section{VipB residue D333 is important for contraction in-vivo}

Interactions of charged residues were previously suggested to be important for contraction of T4

57 phage sheath and R-type pyocin sheath [18,21]. Analysis of interfaces that are expected to be present only in the contracted form of the T6SS sheath of V. cholerae suggested that VipB residues K223 and D333 located on two different VipB-VipB interfaces, one between protomers 
61 significantly contribute to the stability of the contracted structure [22]. This suggests that energy

62 released by forming these interactions could be contributing to driving the sheath contraction.

63 To test this hypothesis, we mutated K223 and D333 to alanine and expressed the mutated vipB

64 in a vipA-msfGFP background to allow for monitoring of sheath dynamics in live-cells. K223A

65 mutation impaired assembly of the sheath and no elongated sheath were observed (Fig. 1b). This

66 was also reflected in the inability of the VipB-K223A mutant to kill target cells (Fig. 1a).

67 Interestingly, VipB-D333A mutant assembled into long non-dynamic sheath structures that were stable in-vivo over more than one hour of imaging (Fig. 1c and Supplementary Video S1 and S2).

69 The number of sheath structures per cell was comparable to the number of structures assembled

70 in wild-type cells suggesting that D333 residue is not critical for sheath assembly. Importantly,

71 VipB-D333A mutation completely blocked target cell killing (Fig. 1a).

\section{VipA linker is critical for sheath contraction in-vivo}

74 Recent atomic models of T6SS sheaths in a contracted state and structures of the R-type pyocin

75 in an extended and contracted state identified intermolecular linkers important for sheath

76 function [20-22]. Interestingly, the N-terminal linker of the R-type pyocin sheath is more

77 stretched in the contracted sheath than in the extended sheath (Supplementary Fig. 1). T4 phage

78 sheath was suggested to contract from the baseplate in a wave of sequentially contracting rings

79 [24]. We hypothesized that stretching of the N-terminal sheath linker upon contraction suggests

80 that the contraction of a basal ring of sheath results in pulling on the VipA N-terminal linker of

81 the next ring, which in turn triggers its contraction. Such a mechanism would lead to propagation 
82 of contraction through the whole sheath. We decided to test this hypothesis by generating a

83 series of mutant $V$. cholerae T6SS sheaths with longer VipA linkers that potentially hinder

84 propagation of contraction. We inserted 1-7 amino acids of the native "AEVELPL" sequence of

85 the linker after the residue 25 of VipA wild-type protein (labeled here as VipA-N1 to VipA-N7). To

86 monitor the assembly and contraction, we fused the VipA and its variants to msfGFP and

87 expressed it from pBAD24 plasmid in the absence of chromosomal vipA [1,22].

All mutant T6SS sheaths assembled with a frequency similar to the wild-type sheath (Fig. 2b), however, frequency of sheath contraction was strongly dependent on the linker length (Fig. 2b, Supplementary Video S3). Whereas insertion of one amino acid (VipA-N1) had almost no effect on sheath dynamics, an elongation by two or more amino acids (VipA-N2-7) reduced the fraction of sheaths that contract during 5 minutes from $50 \%$ (of 159 structures counted, 85 contracted) to $0 \%$ (of 204 structures counted, none contracted). Many of these mutant sheaths were stable over one hour of imaging (Fig. 2c and Supplementary Video S4). Sheaths that occasionally broke after extensive bending caused by movement and growth of cells were however quickly disassembled (Supplementary Video S4). VipA with "AGAGA" sequence inserted, labeled as VipA-

$97 \mathrm{~N} 5(\mathrm{GA})$, also assembled stable full-length sheaths (Fig. 2b, Supplementary Video S3), suggesting that VipA linker length, but not its sequence, is specifically critical for sheath stability. Furthermore, the killing of E. coli MG1655 by V. cholerae T6SS (Fig. 2a) was strongly dependent on the length of the VipA linker. Whereas an extension of this linker by one amino acid had no effect on the killing efficiency, an elongation by two amino acids completely abolished the killing of target cells. 


\section{Stable sheaths assemble from baseplate and around Hcp}

105 To analyze the stable mutant sheaths in more detail, we isolated them by using an approach

106 similar to the one used for the isolation of the wild-type contracted sheath $[1,22]$. Mutant sheaths

107 were expressed in non-flagellated $V$. cholerae strain, cells were lysed and sheaths were purified

108 from soluble proteins and cell debris using ultra-centrifugation. The isolated sheaths were

109 analyzed by negative staining electron microscopy.

110 Analysis of VipB-D333A sheath sample revealed partially fragmented hollow structures with an

111 outer diameter of $260 \mathrm{~nm}$, thus resembling contracted sheaths (Fig. 1d). This suggests that during

112 isolation the VipB-D333A sheaths contract and the D333A mutation destabilizes the contracted

113 structure and this leads to partial fragmentation. Similarly, VipA-N2 sheaths closely resembled

114 the wild-type contracted sheaths as they appeared hollow, had the inner diameter of $100 \AA$ and

115 the outer diameter of $260 \AA$ (Fig. 3a, b). Interestingly, VipA-N3, VipA-N5 and VipA-N5(GA) mutant

116 sheath diameters were $\approx 200 \AA$ and thus narrower than wild-type (Fig. 3a, b). Importantly, uranyl

117 acetate stain was clearly unable to penetrate the sheaths, suggesting that VipA-N3, VipA-N5 and

118 VipA-N5(GA) mutant sheaths were filled with additional protein (Fig. 3a, b).

119 To identify the proteins that were associated with the mutant sheaths, we purified the VipA-

$120 \mathrm{~N} 1,2,3,5$ and $5(\mathrm{GA})$. Proteins in the purified sample were identified using mass spectrometry

121 analysis (Supplementary Table 1). Besides VipA and VipB proteins, VipA-N3, VipA-N5 and VipA-

$122 \mathrm{~N} 5(\mathrm{GA})$ sheath preparations contained large amounts of $\mathrm{Hcp}$, as further confirmed by western-

123 blot (Fig. 3c, lower panel). The presence of Hcp in these mutant sheath samples explains the solid 
appearance on negative stain EM (Fig. 3a). Interestingly, components of T6SS baseplate (TssE, TssF, TssG and TssK) as well as VgrG tip components were also identified in VipA-N3, VipA-N5 and VipA-N5(GA) sheath samples (Supplementary Table 1).

\section{Mutant sheaths assemble only from functional baseplate and non-contractile phenotype is} dominant.

To exclude the possibility that the non-contractile sheaths are aberrant polymers that assemble independently of other T6SS components, we imaged their assembly in a strain that lacks baseplate component TssE. In agreement with the previous observations that TssE is required for efficient sheath assembly $[1,25]$, the frequency of wild-type T6SS sheath assembly decreased by 140-fold in the absence of tssE. Similarly, the assembly of D333A or VipA-N5 sheaths was clearly dependent on the presence of a functional baseplate since the number of structures assembled in the cells lacking tssE was reduced by at least 100 -fold (Fig. 4). This indicates that stable mutant sheaths assemble from a baseplate similarly to the wild-type sheaths.

To test if the mutant sheath subunits can block T6SS activity also in the presence of the wild-type subunits, we induced expression of the VipA-N5 mutant from pBAD24 plasmid in a strain expressing wild-type sheath from the chromosome and measured efficiency of E. coli killing. Low level induction of VipA-N5 by $0.01 \%$ arabinose decreased T6SS-dependent killing of E. coli by 100 fold. The T6SS activity was almost completely blocked by increasing the concentration of arabinose to $0.1 \%$ (Fig. 5) indicating that the ratio of wild-type VipA to VipA-N5 is important for effector delivery. No such inhibition was observed when wild-type VipA was expressed from the 
145 plasmid (Fig. 5). Similarly, low level expression of VipB-D333A decreased T6SS activity by 100-fold

146 and high level of expression blocked the T6SS activity completely (Fig. 5). This dominant negative

147 phenotype suggests that the mutant subunits are structurally compatible with the wild-type

148 subunits, co-assemble into the same structures and thus block T6SS function.

\section{Discussion}

151 T6SS is a highly dynamic system and this complicates detailed biochemical and biophysical

152 characterization of its mode of action. Here we show that the system can be locked in the pre-

153 contraction state by mutagenesis of the linker connecting sheath subunits or changing

154 interactions contributing to the stability of the contracted state. Importantly, some non-

155 contractile sheaths are stable during isolation from cells, assemble around Hcp tube, associate

156 with many T6SS baseplate components, and co-assemble with wild-type extended sheath. This

157 suggests that the structure of non-contractile sheaths is very similar, if not identical, to the wild-

158 type sheath. Moreover, similarly to the wild-type extended sheath, the non-contractile sheaths

159 form in the presence of $\mathrm{ClpV}$ and are therefore different from the previously described

160 polysheath-like structures, which form independently of other T6SS components but only in the

161 absence of ClpV [8].

162 Early electron micrographs of partially contracted T4 phage particles suggested that sheath

163 contraction progresses in a wave of contracting sheath rings from the baseplate towards the

164 phage head [24]. However, it is currently unclear how contraction of one sheath ring triggers

165 contraction of the next ring. As we show here, insertion of two residues into the VipA N-terminal 
166 linker prevents T6SS sheath contraction in-vivo, suggesting that the exact length of the linker

167 connecting the subunits is essential for sheath contraction initiation or propagation of the 168 contraction along the sheath (Fig. 6 and Supplementary Fig. 1). T6SS, T4 and R-type pyocin sheath

169 structures identified charged residues important for stability of contracted structures [18,21,22].

170 Many of these interactions specifically form during contraction and were thus proposed to be

171 driving sheath contraction $[18,21]$. Together with our data, this suggests that the energy gained

172 by formation of new charge interactions is potentially used to pull on the linkers between sheath

173 subunits and thus propagating the contraction to the next sheath ring.

174 Interestingly, the stable non-contractile sheaths also associated with many baseplate 175 components unlike contracted sheaths (Supplementary Table S1) [1,22]. This suggests that after 176 sheath contraction the baseplate is destabilized and dissociates from the sheath. Live-cell

177 imaging of sheath dynamics and localization indeed showed that few seconds after contraction,

178 but before disassembly, sheaths often dissociate from the initial cell envelope attachment site

179 [15]. This is consistent with the observation that T6SS assemble repeatedly inside cells and the 180 components of the baseplate are likely reused for new rounds of assembly. In related contractile 181 nanomachines, which are only used once, the contracted sheaths remain stably associated with 182 the baseplates and likely provide mechanical stability to the contracted particles. In the case of 183 contractile phages, the sheath connects baseplate and the phage head as the DNA is translocated 184 and in the case of R-type pyocin, the sheath might be needed to stabilize the tube, which allows 185 ion leakage and the killing of target bacterial cell $[9,21,26]$. 
186 The approach used here to stabilize the pre-contraction state of T6SS will be likely invaluable for

187 further attempts to dissect T6SS mode of action at the molecular level in various bacteria and

188 may be also used to study related contractile nanomachines with a major relevance for viral

189 infection, bacterial competition and pathogenicity.

190

191

192 Material Methods

\section{Bacterial strains and DNA manipulations}

194 V. cholerae 2740-80 parental, vipA-msfGFP, $\Delta v i p A, \Delta v i p B, \Delta t s s E$ strains and the pBAD24-vipA195 sfGFP plasmid were described previously [1,22]. vipA mutants on pBAD24 plasmid were 196 generated using standard techniques. Mutant vipA genes encode "A, AE, AEV, AEVE, AEVEL, 197 AGAGA, AEVELP or AEVELPL" residues inserted right after residue 25 of wild-type vipA. The 198 insertions represent either duplication of the native sequence or a sequence encoding "AGAGA". 199 V. cholerae 2740-80 $\triangle$ vipA-vipB strain was created by replacing vipA and vipB with a gene 200 encoding "MSKEGSVGRLDQA" peptide (first seven residues of vipA and last six residues of vipB

201 fused in frame) and $V$. cholerae 2740-80 $\Delta v i p A-v i p B-t s s E$ strain was created by replacing vipA, 202 vipB and tsSE with a gene encoding "MSKEGSVRKYRVF" peptide (first seven residues of vipA and 203 last six residues of tssE fused in frame) by allelic exchange as was done previously. vipB (wt) was 204 cloned into pBAD24 and pBAD33 plasmids using standard techniques. K223A and D333A 205 mutations were introduced into vipB using mutagenic primers. All PCR-generated products were 206 verified by sequencing. Plasmids were transformed into $V$. cholerae by electroporation. 
207

208

209

210

211

212

213

214

215

216

217

218

219

220

221

222

223

224

225

226

227

Gentamicin-resistant E. coli MG1655 strain with pUC19 plasmid was used in bacterial killing assays. Antibiotic concentrations used were streptomycin $(50 \mu \mathrm{g} / \mathrm{ml})$, ampicillin $(200 \mu \mathrm{g} / \mathrm{ml})$, chloramphenicol $(20 \mu \mathrm{g} / \mathrm{ml})$ and gentamicin $(15 \mu \mathrm{g} / \mathrm{ml})$. Lysogeny broth (LB) was used for all growth conditions. Liquid cultures were grown aerobically at $37^{\circ} \mathrm{C}$.

\section{Bacterial killing assay}

V. cholerae $2740-80$ strains as indicated and E. coli MG1655 with empty pUC19 plasmid were incubated overnight at $37^{\circ} \mathrm{C}$ in LB supplemented with appropriate antibiotics. Cultures were diluted 100 -fold, and grown to OD 0.8-1.2 in presence of appropriate antibiotics and $0.01 \%$ arabinose for strains with pBAD plasmids. Cells were washed and mixed at final OD of $\approx 10$ in 10:1 ratio ( $V$. cholerae to $E$. coli) as specified, and $5 \mu$ of the mixture was spotted on a pre-dried LB agar plate containing $0.01 \%$ arabinose and ampicillin or no antibiotic. After $3 \mathrm{~h}$, bacterial spots were cut out and the cells were re-suspended in $0.5 \mathrm{ml} \mathrm{LB}$. The cellular suspension was serially diluted (1:10) in LB, and $5 \mu$ of the suspensions were spotted on selective plates (gentamicin for E. coli and streptomycin for $V$. cholerae). Colonies were counted after $\approx 16 \mathrm{~h}$ incubation at $30^{\circ} \mathrm{C}$. Two or more biological replicates were analyzed.

\section{Fluorescence microscopy}

Procedures similar to those described previously [15] were used to detect fluorescence signal in $V$. cholerae. Overnight cultures of $V$. cholerae carrying pBAD24 plasmid with the respective inserts were diluted 100-fold into fresh LB supplemented with ampicillin, streptomycin, and $0.01 \%$ or $0.03 \%$ arabinose and cultivated for $2.5-3.0 \mathrm{~h}$ to optical density (OD) at $600 \mathrm{~nm}$ of about 
$0.8-1.2$. Cells from $1 \mathrm{ml}$ of the culture were re-suspended in $\approx 50 \mu \mathrm{LB}$ (to OD $\approx 20$ ), spotted on a thin pad of $1 \%$ agarose in LB, and covered with a glass coverslip. Cells were immediately imaged at room temperature. A previously described microscope setup was used [22]. VisiView software (Visitron Systems, Germany) was used to record images. Fiji [27] was used for all image analysis and manipulations as described previously [28]. Bleach correction was used if necessary [29]. Contrast on compared sets of images was adjusted equally. All imaging experiments were performed with three biological replicates.

\section{VipA/VipB sheath preparation}

Overnight cultures of the indicated strains were diluted 1:1000 in 0.5 I of fresh LB supplemented with appropriate antibiotics, and then shaken at $37^{\circ} \mathrm{C}$ and $250 \mathrm{rpm}$ to an OD of $\approx 1.2$. Cells were centrifuged for $20 \mathrm{~min}$ at $5000 \mathrm{xg}$ and $4^{\circ} \mathrm{C}$, re-suspended in $20 \mathrm{ml} \mathrm{PBS}$ and centrifuged again for

242 thawed, re-suspended in $20 \mathrm{ml}$ of TN-buffer $(20 \mathrm{mM}$ Tris, $150 \mathrm{mM} \mathrm{NaCl}, \mathrm{pH} 8.3$ ) and lysed by

243 addition of $(0.75 \mathrm{x})$ CelLytic $^{\mathrm{TM}} \mathrm{B}$, Lysozyme $(200 \mu \mathrm{g} / \mathrm{ml})$, EDTA $(5 \mathrm{mM})$ and incubation at $37^{\circ} \mathrm{C}$.

244 DNase $(50 \mu \mathrm{g} / \mathrm{ml})$ and $\mathrm{MgCl}_{2}(10 \mathrm{mM})$ was added to cleave DNA. After 15 min incubation at $37^{\circ} \mathrm{C}$,

245 cell debris was removed by centrifugation for $20 \mathrm{~min}$ at 10,000 x g. Cleared supernatants were 246 subjected to ultraspeed centrifugation for $1.5 \mathrm{~h}$ at $104,000 \times \mathrm{g}$ and $4^{\circ} \mathrm{C}$ and the resulting pellet 247 was washed with $1 \mathrm{ml} \mathrm{TN}$-buffer and subsequently re-suspended in $1 \mathrm{ml} \mathrm{TN}$-buffer, insoluble 248 material was removed by centrifugation for $1 \mathrm{~min}$ at 10,000 $\mathrm{xg}$. The supernatant was subjected 249 to another round of ultraspeed centrifugation for $1 \mathrm{~h}$ at $104,000 \times \mathrm{g}$ and $4^{\circ} \mathrm{C}$ and the resulting 
250

pellet was re-suspended in $70 \mu \mathrm{l}$ of TN-buffer for further analysis. Purity of the sample was assessed by Coomassie stained SDS-PAGE.

\section{Mass spectrometry}

Sheath samples (30-100 $\mu \mathrm{g}$ ) were reduced with $5 \mathrm{mM}$ tris(2-chlorethyl)phosphate, shaking for $1 \mathrm{~h}$ at $37^{\circ} \mathrm{C}$ and alkylated with $10 \mathrm{mM}$ iodoacetamide, shaking for $30 \mathrm{~min}$ at $25^{\circ} \mathrm{C}$ in the dark. Proteins were digested using sequencing-grade modified trypsin (1/250, w/w; Promega, USA) overnight at $37^{\circ} \mathrm{C}$. After digestion, the samples were supplemented with TFA to a final concentration of 1\%. Peptides were desalted on C18 reversed phase spin columns according to the manufacturer's instructions (Microspin, Harvard Apparatus), dried under vacuum and re-suspended in LC-MS buffer $(0.15 \%$ formic acid, $2 \%$ acetonitrile in HPLC water) at $\approx 0.5 \mathrm{mg} / \mathrm{ml} .1 \mu \mathrm{g}$ of peptides of each sample were subjected to LC-MS analysis using a dual pressure LTQ-Orbitrap Elite mass spectrometer connected to an electrospray ion source (both Thermo Fisher Scientific) as described recently [30] with a few modifications. In brief, peptide separation was carried out using an EASY nLC-1000 system (Thermo Fisher Scientific) equipped with a RP-HPLC column $(75 \mu \mathrm{m} \times 30 \mathrm{~cm})$ packed in-house with C18 resin (ReproSil-Pur C18-AQ, $1.9 \mu \mathrm{m}$ resin; Dr. Maisch $\mathrm{GmbH}$, Ammerbuch-Entringen, Germany) using a linear gradient from 95\% solvent A $(0.15 \%$ formic acid, $2 \%$ acetonitrile) and $5 \%$ solvent B ( $98 \%$ acetonitrile, $0.15 \%$ formic acid) to $28 \%$ solvent B over $75 \mathrm{~min}$ at a flow rate of $0.2 \mu \mathrm{l} / \mathrm{min}$. The data acquisition mode was set to obtain one high resolution MS scan in the FT part of the mass spectrometer at a resolution of 240000 full width at half maximum (at $\mathrm{m} / \mathrm{z} 400$ ) followed by MS/MS scans in the linear ion trap of the 20 most intense ions. The charged state screening modus was enabled to exclude unassigned and 
272 singly charged ions and the dynamic exclusion duration was set to $20 \mathrm{~s}$. The ion accumulation

273 time was set to $300 \mathrm{~ms}(\mathrm{MS})$ and $50 \mathrm{~ms}$ (MS/MS). The collision energy was set to $35 \%$, and one

274 microscan was acquired for each spectrum. For all LC-MS measurements, singly charged ions and

275 ions with unassigned charge state were excluded from triggering MS2 events. The generated raw

276 files were imported into the Progenesis LC-MS software (Nonlinear Dynamics, Version 4.0) and

277 analyzed using the default settings. MS/MS-data were exported directly from Progenesis LC-MS

278 in mgf format and searched against a decoy database of the forward and reverse sequences of

279 the predicted proteome from Vibrio cholerae (Uniprot, Organism ID: 243277, download date:

$28007 / 11 / 2016$, total of 3784 entries) using MASCOT. The search criteria were set as following: full

281 tryptic specificity was required (cleavage after lysine or arginine residues); 3 missed cleavages

282 were allowed; carbamidomethylation (C), was set as fixed modification; oxidation (M) as variable

283 modification. The mass tolerance was set to $10 \mathrm{ppm}$ for precursor ions and $0.6 \mathrm{Da}$ for fragment

284 ions. Results from the database search were imported into Progenesis and the protein false

285 discovery rate (FDR) was set to $1 \%$ using the number of reverse hits in the dataset. The final

286 protein lists containing the summed peak areas of all identified peptides for each protein were

287 exported from Progenesis LC-MS and further statistically analyzed using an in-house developed

288 R script (SafeQuant) [30]. For relative quantification of protein abundances between sheath 289 samples, MS1 peak intensities of the query protein (Hcp, VC_A1415; VgrG3, VC_A0123; TssE,

290 VC_A0109; TssF, VC_A0110; TssG, VC_A0111; TssK, VC_A0114) were normalized by division with wild-type sample. Two biological replicates were analyzed. 


\section{Negative stain electron microscopy}

295

296

297

298

299

300

301

302

303

304

305

306

307

308

309

310

311

312

313

314

315

300-mesh copper grids were glow-discharged for $20 \mathrm{~s}$, samples $(5 \mu \mathrm{l}$, protein concentration aprox.

$0.1 \mu \mathrm{g} / \mathrm{ml}$ ) were adsorbed for $1 \mathrm{~min}$ and blotted using Whatman \#1 filter paper. The grids were washed five times with $\mathrm{H}_{2} \mathrm{O}$, and once using $2 \%$ uranyl acetate, followed by a 20 -s staining with 2\% uranyl acetate. Grids were imaged on a CM-100 microscope (Philips N.V., Amsterdam, Netherlands) equipped with a Veleta $2 \mathrm{k} \times 2 \mathrm{k}$ camera (Olympus K.K., Tokio, Japan) at $80 \mathrm{kV}$ and a magnification of 64,000x. The pixel size was $7.4 \AA$ A . Fiji [27] was used for all image analysis.

\section{Immunoblot analysis}

5-10 $\mu \mathrm{l}$ of purified sheath samples were mixed with 1.2-2.4 $\mu \mathrm{l} 4 \mathrm{x}$ NuPAGE ${ }^{\circledR}$ LDS Sample Buffer (Life Technologies). Samples were incubated for $10 \mathrm{~min}$ at $95^{\circ} \mathrm{C}$, centrifuged, cooled and $2 \mu \mathrm{l} 1 \mathrm{M}$ DTT was added. Samples were heated again for $10 \mathrm{~min}$ at $72^{\circ} \mathrm{C}$, centrifuged, loaded on $10 \%$ polyacrylamide gels and transferred to nitrocellulose membrane (Amersham Biosciences, UK).

Membrane was blocked with $5 \%$ milk in Tris buffered saline $(\mathrm{pH} \mathrm{7.4)} \mathrm{containing} \mathrm{Tween} 0.1 \%$ (TBST), incubated with primary peptide antibody against Hcp (“QSGQPSGQRVHKPF”, Genscript, USA [1]), or peptide antibody against VipB, (“QENPPADVRSRRPL", Genscript, USA [22]) for $16 \mathrm{hr}$ at $4^{\circ} \mathrm{C}$ or $1 \mathrm{hr}$ at room temperature, washed with TBST, incubated for $1 \mathrm{hr}$ with horseradish peroxidase-labeled anti-rabbit antibody (Jackson ImmunoResearch Inc., USA), and washed with the recommended buffer, and peroxidase was detected by LumiGLO ${ }^{\circledR}$ Chemiluminescent Substrate (KPL, Inc., Gaithersburg, Maryland, USA). Nitrocellulose membrane was stripped using Restore $^{\mathrm{TM}}$ Western Blot Stripping Buffer (Thermo Scientific, USA) and reprobed using the same protocol. 
317 Molecular analysis

318 Structures of PA0622 in the contracted state (PDB ID: 3J9R) [21] and of VipA and VipB in the

319 contracted state (PDB ID: 3J9G) [22] were aligned based on their 3D-structure using UCSF

320 Chimera [31].

321

322

\section{Author contributions}

$324 \mathrm{M} . \mathrm{Br}$. generated and characterized the mutant sheaths. Isolated and purified the sheaths, 325 performed negative stain electron microscopy, mass-spectrometry and western-blot analysis of 326 their structure and composition. J.W. contributed to electron microscopy data analysis. M.Ba.

327 conceived the project and analyzed the data. M.Br., J.W. and M.Ba. wrote the manuscript. All 328 authors read the manuscript.

332 The work was supported by Swiss National Science Foundation (SNSF) grant 31003A_159525 and

333 the University of Basel. We acknowledge the Biozentrum proteomics core facility for mass 334 spectrometry measurements. 


\section{References}

1. Basler M, Pilhofer M, Henderson GP, Jensen GJ, Mekalanos JJ (2012) Type VI secretion requires a dynamic contractile phage tail-like structure. Nature 483: 182-186.

2. Hood RD, Singh P, Hsu F, Güvener T, Carl MA, Trinidad RRS, Silverman JM, Ohlson BB, Hicks KG, Plemel RL, et al. (2010) A type VI secretion system of Pseudomonas aeruginosa targets a toxin to bacteria. Cell Host Microbe 7: 25-37.

3. Maclntyre DL, Miyata ST, Kitaoka M, Pukatzki S (2010) The Vibrio cholerae type VI secretion system displays antimicrobial properties. Proc Natl Acad Sci U S A 107: 19520-19524.

4. Mougous JD, Cuff ME, Raunser S, Shen A, Zhou M, Gifford CA, Goodman AL, Joachimiak G, Ordoñez CL, Lory S, et al. (2006) A virulence locus of Pseudomonas aeruginosa encodes a protein secretion apparatus. Science 312: 1526-1530.

5. Pukatzki S, Ma AT, Sturtevant D, Krastins B, Sarracino D, Nelson WC, Heidelberg JF, Mekalanos JJ (2006) Identification of a conserved bacterial protein secretion system in Vibrio cholerae using the Dictyostelium host model system. Proc Natl Acad Sci U A 103: 1528-1533.

6. Arisaka F, Tschopp J, van Driel R, Engel J (1979) Reassembly of the bacteriophage T4 tail from the core-baseplate and the monomeric sheath protein P18: A co-operative association process. J Mol Biol 132: 369-386.

7. Brunet YR, Henin J, Celia H, Cascales E (2014) Type VI secretion and bacteriophage tail tubes share a common assembly pathway. EMBO Rep 15: 315-321.

8. Kapitein N, Bonemann G, Pietrosiuk A, Seyffer F, Hausser I, Locker JK, Mogk A (2013) ClpV recycles VipA/VipB tubules and prevents non-productive tubule formation to ensure efficient type VI protein secretion. Mol Microbiol 87: 1013-1028. 
9. Leiman PG, Shneider MM (2012) Contractile tail machines of bacteriophages. Adv Exp Med Biol 726: 93-114.

10. Tschopp J, Arisaka F, van Driel R, Engel J (1979) Purification, characterization and reassembly of the bacteriophage T4D tail sheath protein P18. J Mol Biol 128: 247-258.

11. Brunet YR, Zoued A, Boyer F, Douzi B, Cascales E (2015) The Type VI Secretion TssEFGK-VgrG PhageLike Baseplate Is Recruited to the TssJLM Membrane Complex via Multiple Contacts and Serves As Assembly Platform for Tail Tube/Sheath Polymerization. PLoS Genet 11: e1005545.

12. Durand E, Nguyen VS, Zoued A, Logger L, Péhau-Arnaudet G, Aschtgen M-S, Spinelli S, Desmyter A, Bardiaux B, Dujeancourt A, et al. (2015) Biogenesis and structure of a type VI secretion membrane core complex. Nature 523: 555-560.

13. Planamente S, Salih O, Manoli E, Albesa-Jové D, Freemont PS, Filloux A (2016) TssA forms a gp6-like ring attached to the type VI secretion sheath. EMBO J 35: 1613-1627.

14. Zoued A, Durand E, Brunet YR, Spinelli S, Douzi B, Guzzo M, Flaugnatti N, Legrand P, Journet L, Fronzes R, et al. (2016) Priming and polymerization of a bacterial contractile tail structure. Nature 531: 59-63.

15. Basler M, Mekalanos JJ (2012) Type 6 secretion dynamics within and between bacterial cells. Science 337: 815 .

16. Brunet YR, Espinosa L, Harchouni S, Mignot T, Cascales E (2013) Imaging Type VI Secretion-Mediated Bacterial Killing. Cell Rep 3: 36-41. 
18. Aksyuk AA, Leiman PG, Kurochkina LP, Shneider MM, Kostyuchenko VA, Mesyanzhinov VV, Rossmann MG (2009) The tail sheath structure of bacteriophage T4: a molecular machine for infecting bacteria. EMBO J 28: 821-829.

19. Aksyuk AA, Kurochkina LP, Fokine A, Forouhar F, Mesyanzhinov VV, Tong L, Rossmann MG (2011) Structural conservation of the myoviridae phage tail sheath protein fold. Struct Lond Engl 1993 19: 1885-1894.

20. Clemens DL, Ge P, Lee B-Y, Horwitz MA, Zhou ZH (2015) Atomic structure of T6SS reveals interlaced array essential to function. Cell 160: 940-951.

21. Ge P, Scholl D, Leiman PG, Yu X, Miller JF, Zhou ZH (2015) Atomic structures of a bactericidal contractile nanotube in its pre- and postcontraction states. Nat Struct Mol Biol 22: 377-382.

22. Kudryashev M, Wang RY-R, Brackmann M, Scherer S, Maier T, Baker D, DiMaio F, Stahlberg H, Egelman EH, Basler M (2015) Structure of the Type VI Secretion System Contractile Sheath. Cell 160: 952-962.

23. Kube S, Kapitein N, Zimniak T, Herzog F, Mogk A, Wendler P (2014) Structure of the VipA/B type VI secretion complex suggests a contraction-state-specific recycling mechanism. Cell Rep 8: 20-30.

24. Moody MF (1973) Sheath of bacteriophage T4. 3. Contraction mechanism deduced from partially contracted sheaths. J Mol Biol 80: 613-635.

25. Vettiger A, Basler M (2016) Type VI Secretion System Substrates Are Transferred and Reused among Sister Cells. Cell 167: 99-110.e12.

26. Hu B, Margolin W, Molineux IJ, Liu J (2015) Structural remodeling of bacteriophage T4 and host membranes during infection initiation. Proc Natl Acad Sci U S A 112: E4919-E4928. 
28. Basler M, Ho BT, Mekalanos JJ (2013) Tit-for-Tat: Type VI Secretion System Counterattack during Bacterial Cell-Cell Interactions. Cell 152: 884-894.

407 29. Miura K, Rueden C, Hiner M, Schindelin J, Rietdorf J (2014) ImageJ Plugin CorrectBleach V2.0.2.

408 30. Glatter T, Ludwig C, Ahrné E, Aebersold R, Heck AJR, Schmidt A (2012) Large-scale quantitative assessment of different in-solution protein digestion protocols reveals superior cleavage efficiency of tandem Lys-C/trypsin proteolysis over trypsin digestion. J Proteome Res 11: 5145-5156.

411 31. Pettersen EF, Goddard TD, Huang CC, Couch GS, Greenblatt DM, Meng EC, Ferrin TE (2004) UCSF 412 Chimera-A visualization system for exploratory research and analysis. J Comput Chem 25: 16051612.

414 
417 Supplementary Video legends

Supplementary Video S1. VipB residue D333 is crucial for sheath contraction.

419 Time lapse videos of $V$. cholerae vipA-msfGFP vipB-, with pBAD24 plasmid encoding VipB or VipB-

420 D333A mutant. Phase contrast and GFP-channel are merged in the left panel and only the GFP-

421 signal is shown in the right panel. One field of view is $19.5 \times 19.5 \mu \mathrm{m}$.

Supplementary Video S2. VipB residue D333 is crucial for sheath contraction.

424 Long time lapse videos of $V$. cholerae vipA-msfGFP vipB-, with pBAD24 plasmid encoding VipB 425 D333A mutant. Phase contrast and GFP-channel are merged. One field of view is $19.5 \times 19.5 \mu \mathrm{m}$.

428 Time lapse videos of $V$. cholerae vipA', with pBAD24 plasmid encoding indicated VipA-msfGFP

429 linker mutants. Phase contrast and GFP-channel are merged in the left panel and only the GFP-

430 signal is shown in the right panel. One field of view is $19.5 \times 19.5 \mu \mathrm{m}$.

\section{Supplementary Video S4. Length of N-terminal linker of VipA controls sheath contraction.}

433 Long time lapse videos of $V$. cholerae vipA', with pBAD24 plasmid encoding VipA-N5-msfGFP

434 mutant. Phase contrast and GFP-channel are merged. Arrows mark T6SS sheaths that break and 435 subsequently get disassembled. One field of view is $19.5 \times 19.5 \mu \mathrm{m}$. 
a

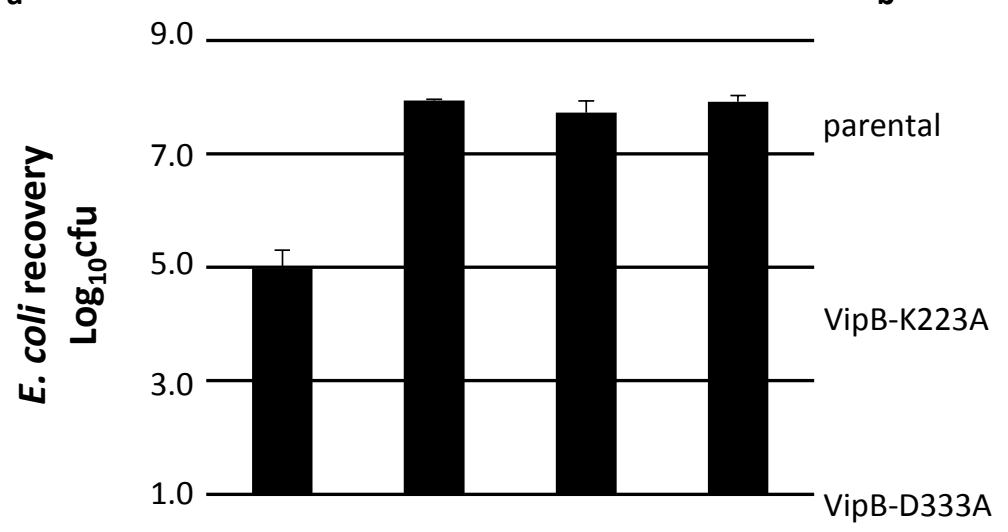

C

\begin{tabular}{llllll}
$v i p A-G F P$ & + & + & + & + & \\
\hline$v i p B$ & + & - & K223A & D333A
\end{tabular}

VipB-D333A
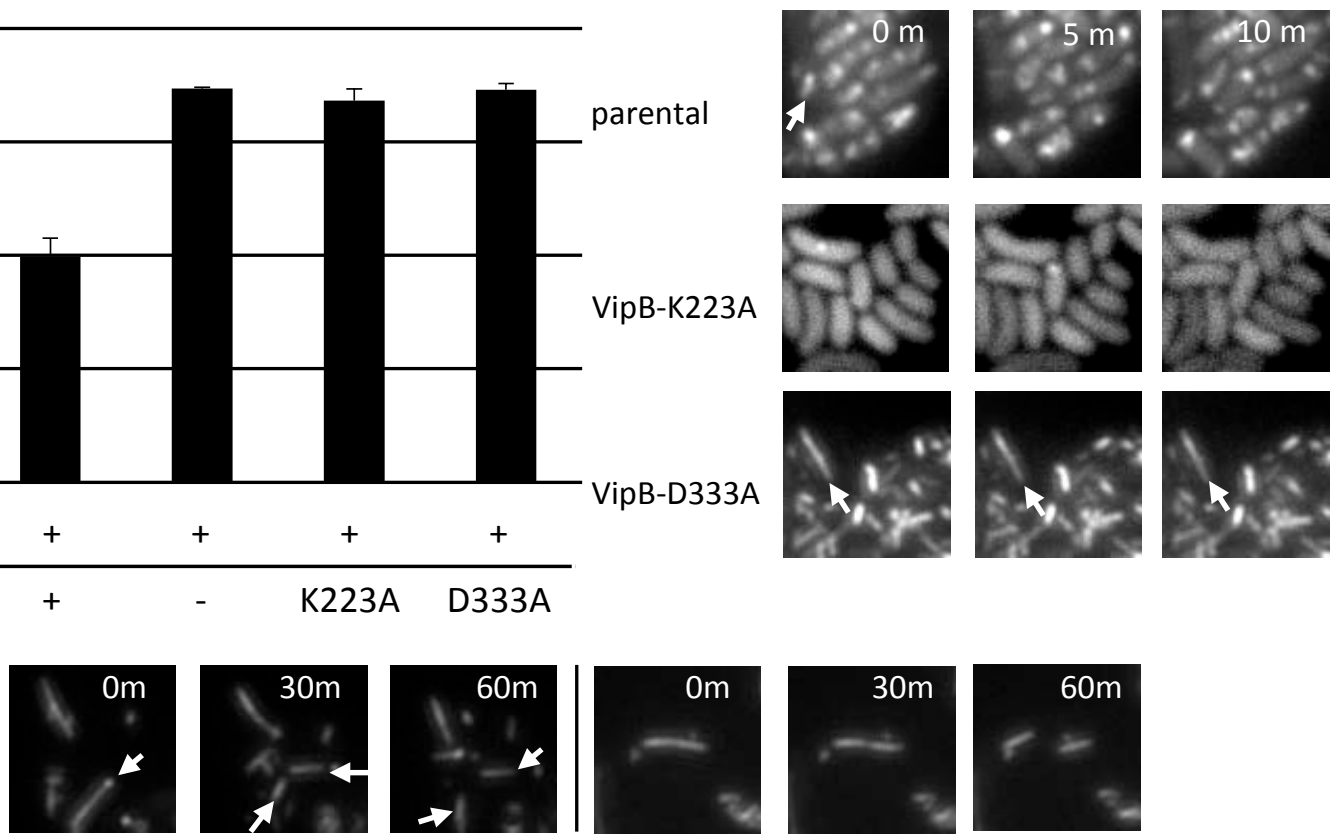

d
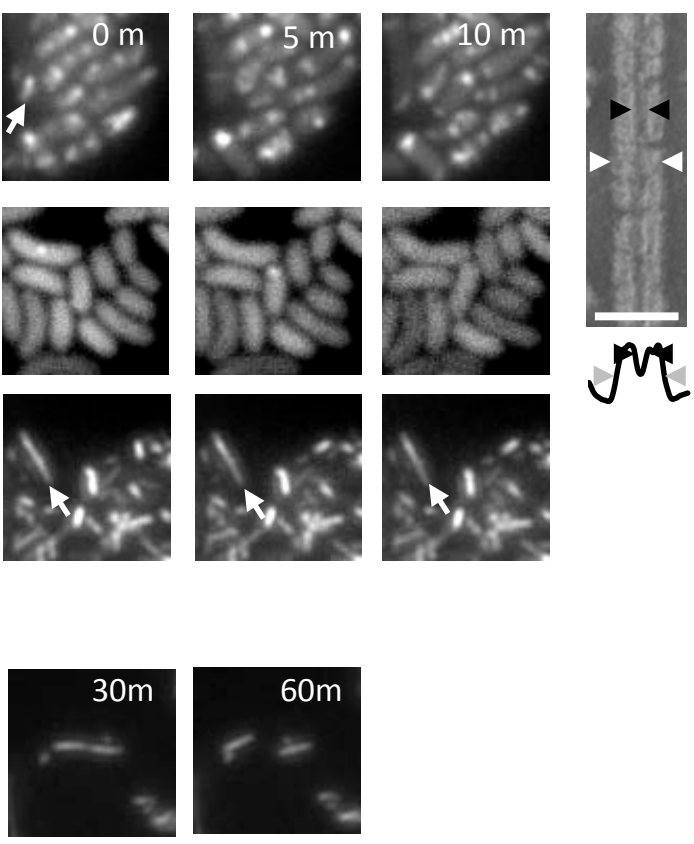

Figure 1 Charged residues of VipB are essential for sheath assembly and contraction

a $E$. coli survival $( \pm \mathrm{SD}, \mathrm{N}=3$ ) after $3 \mathrm{~h}$ competition with indicated $V$. cholerae strains in a $1: 10$ ratio on plate. b Fluorescence timelapse images of $V$. cholerae vipA-msfGFP, vipB- complemented with vipB-K223A, vipB-D333A on pBAD24. c Long timelapses of $V$. cholerae vipA-msfGFP, vipB- complemented with vipB-D333A on pBAD24. d Electron micrograph of purified VipB-D333A sheath and below a plot of the summed intensities. The inner diameter is marked with black arrowheads and the outer diameter with grey arrowheads. 


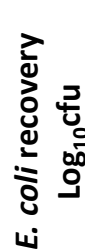

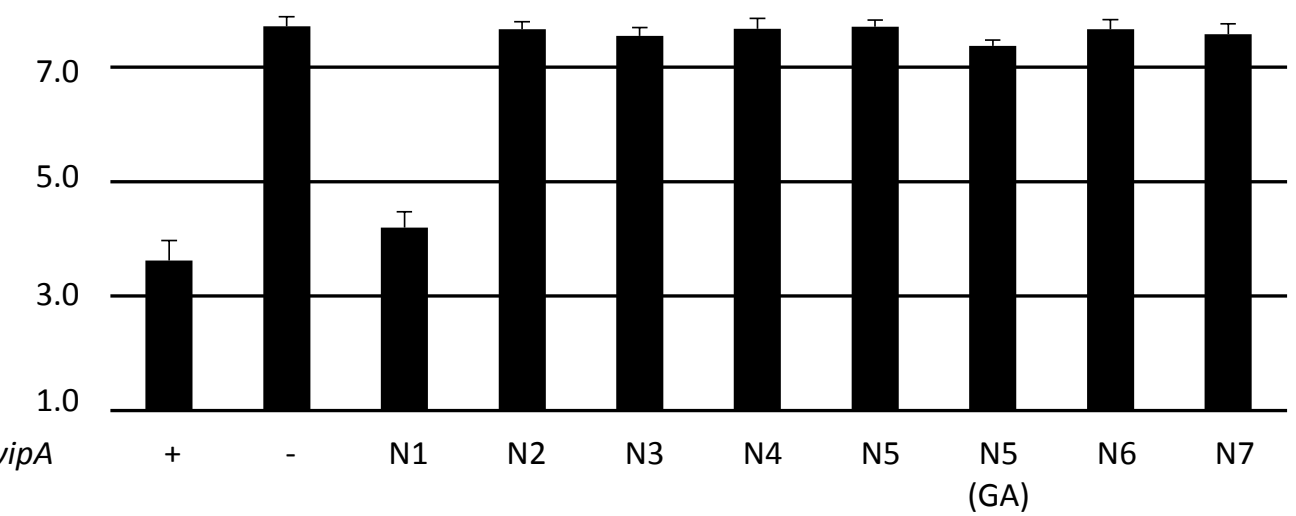

b

wt
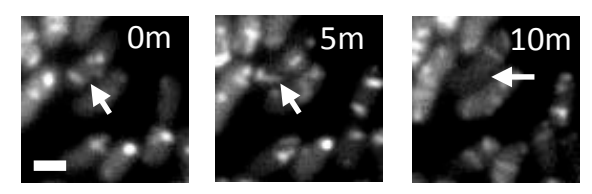

N5
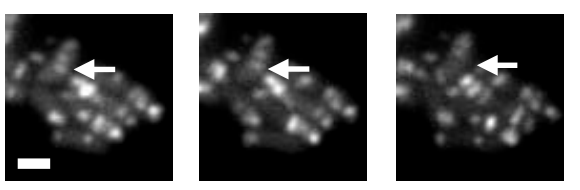

N5 (GA)
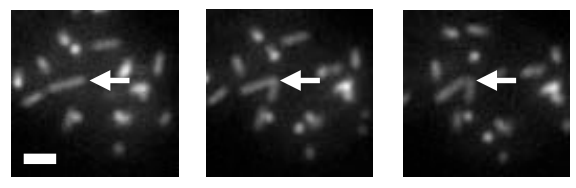

N2
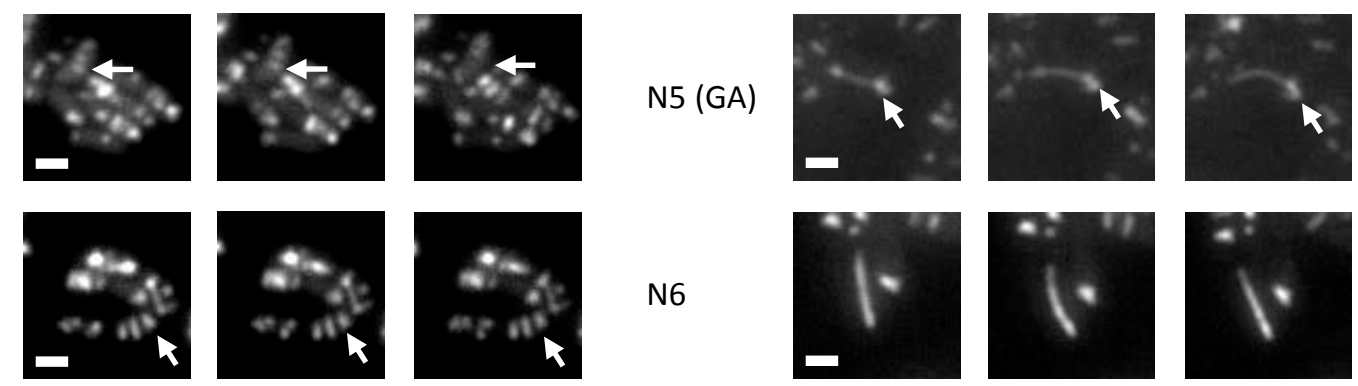

N6
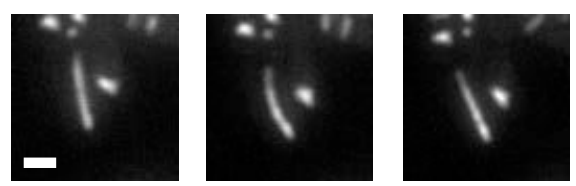

N3
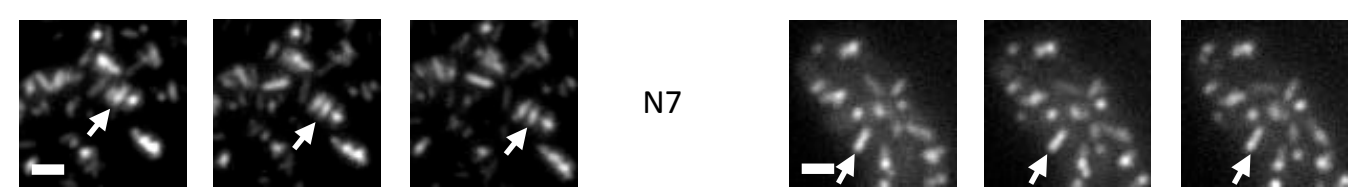

N4
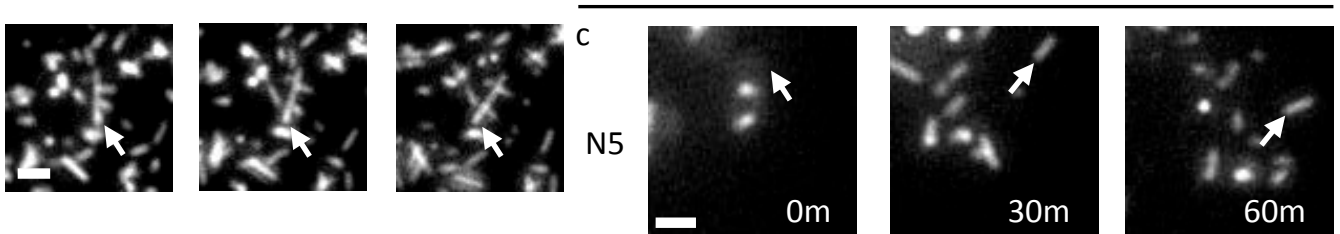

Figure 2 Length of $\mathbf{N}$-terminal linker of VipA controls sheath contraction

a $E$. coli survival $( \pm S D, N=3$ ) after 3 competition with indicated $V$. cholerae strains in a $1: 10$ ratio on plate. b Fluorescence timelapse images of $V$. cholerae vipA- complemented with indicated msfGFPtagged-vipA variants on pBAD24. c Long timelapse of $V$. cholerae vipA- complemented with vipA-N5msfGFP on pBAD24. Scale bars are $1 \mu \mathrm{m}$. 


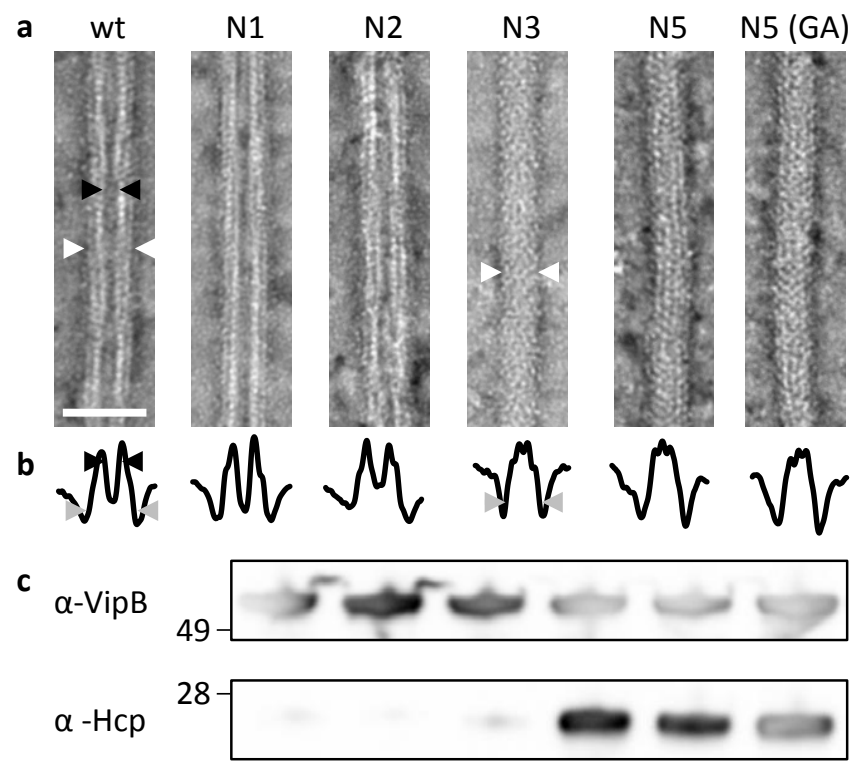

Figure $3 \mathrm{Hcp}$ is enriched in VipA mutants with a linker elongated by three or more amino acids. a Electron micrographs of sheath samples. Wild-type sheath and those with additional one or two amino acids inserted are hollow but with three or more amino acids show a protein density in the center. Black arrowheads mark the inner diameter and white arrowheads mark the outer diameter. Scale bar is $50 \mathrm{~nm}$. b Plot of summed intensities of the micrographs in a. The inner diameter is marked with black arrowheads and the outer diameter with grey arrowheads. c Immunoblots against Hcp and VipB of the samples in a. 
a
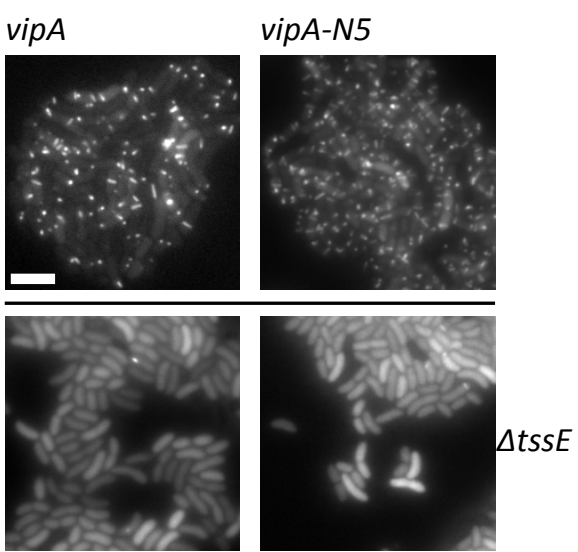

b

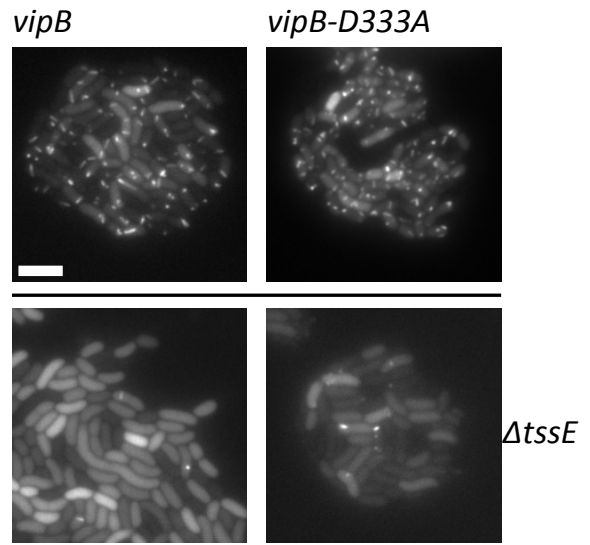

Figure 4 Assembly of non-contractile sheaths depends on presence of TssE

a Fluorescence microscopy images of $\mathrm{V}$. cholerae vipA- or vipA', tSSEcomplemented with vipA-msfGFP or vipA-5aalinker-msfGFP on pBAD24. b

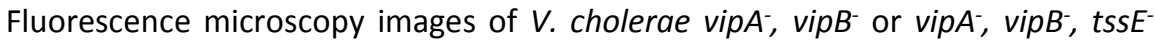
complemented with vipA-msfGFP on pBAD24 and vipB or vipB-D333A on pBAD33. 


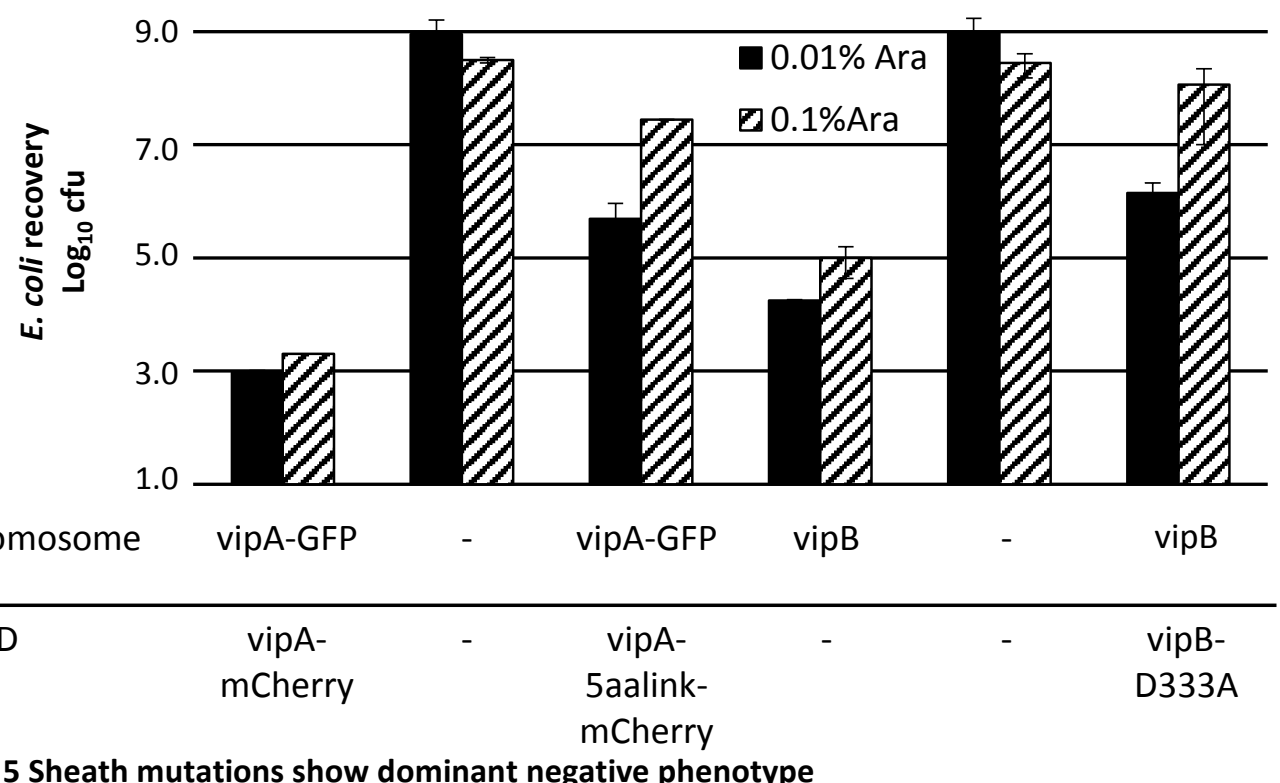

Figure 5 Sheath mutations show dominant negative phenotype

E. coli survival $( \pm \mathrm{SD}, \mathrm{N}=2$ ) after 3 h competition with indicated $V$. cholerae strains in a 1:10 ratio on plate at two different arabinose concentrations. 


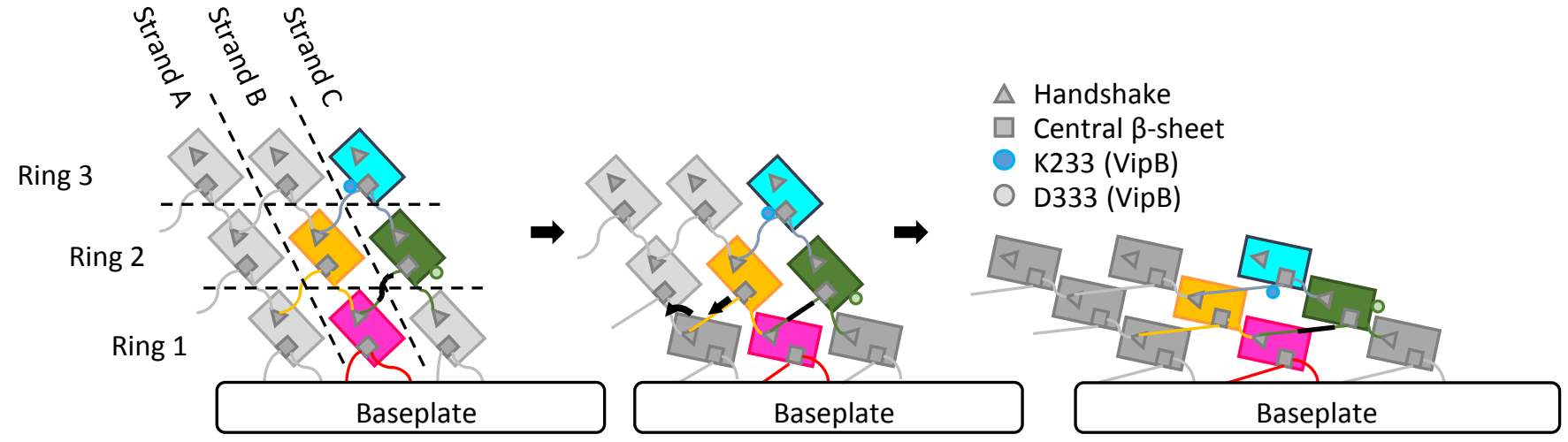

Figure 6 Model of the mechanism of contraction of contractile sheaths

Scheme representing the connections between strands and rings in pyocin and T6SS sheaths. Ring 1 contracts after an initial trigger coming from the baseplate via TsSE. Ring 1 pulls on a linker region (black) of ring 2 and by this propagates the contraction throughout the sheath. 
a

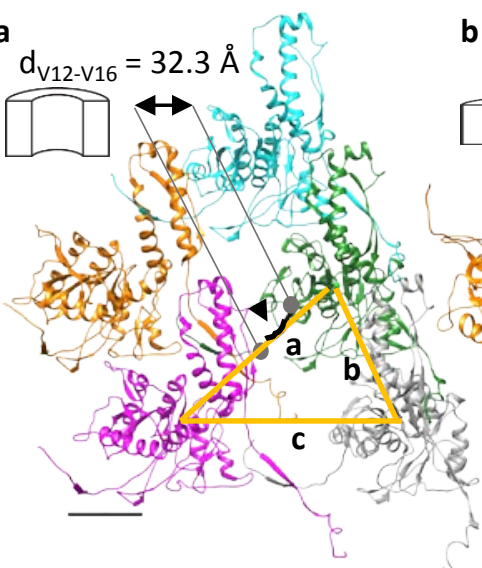

b

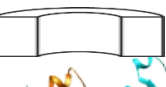

$d_{\mathrm{v} 12-\mathrm{v} 16}=37.3 \AA$

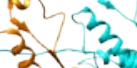

$a=57.8 \AA$
$b=42.9 \AA$
$c=60.6 \AA$

$a=40.0 \AA$

$\mathrm{b}=47.8 \AA$

$c=78.6 \AA$

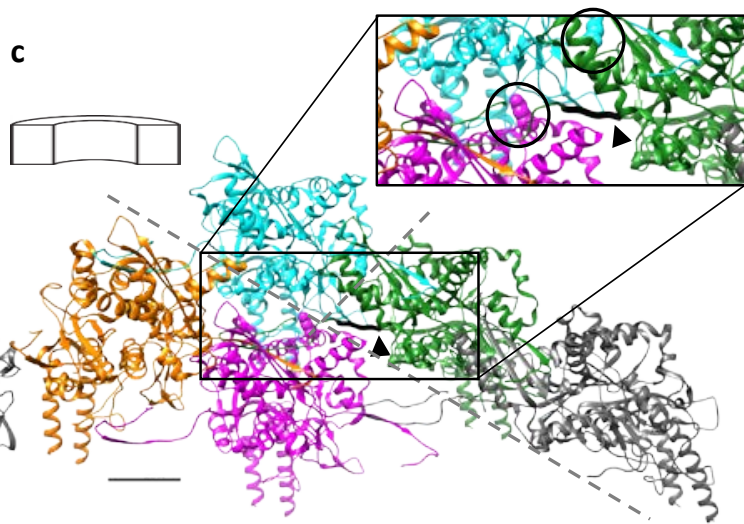

Supplementary Figure 1 Protomers of contractile sheaths are interconnected by linkers that are stretched in the contracted state

a Structure of an R-type pyocin sheath in the extended state (PDB ID: 3J9Q) viewed from inside the sheath. Individual protomers are interlaced via linkers (black, arrowhead) through interactions formed by $\beta$-sheets. $\mathbf{b}$ Structure of an R-type pyocin sheath in the contracted state (PDB ID: 3J9R). The linkers interconnecting (black, arrowhead) two strands are stretched. c Structure of the contracted T6SS-sheath from V. cholerae (PDB ID: 3J9G). The backbone of the linker region of VipA that connects the green protomer with the magenta protomer is shown thick in black (arrowhead). Mutated residues on VipB are highlighted in black circles in the inset. D333 is shown on the cyan protomer, K223 is shown on the magenta protomer Distances between center of masses of different protomers are depicted as orange lines. The scale bars are $20 \AA$. 
Supplementary Table 1 Hcp and Baseplate Components Are Detected by Mass Spectrometry.

Fold change in amounts of indicated T6SS-proteins, normalized to wild-type. Two biological replicates are shown.

\begin{tabular}{|c|c|c|c|c|c|c|c|c|c|c|}
\hline & \multicolumn{2}{|l|}{ N1 } & \multicolumn{2}{|l|}{$\mathrm{N} 2$} & \multicolumn{2}{|l|}{ N3 } & \multicolumn{2}{|l|}{ N5 } & \multicolumn{2}{|l|}{ N5 (GA) } \\
\hline & $\begin{array}{l}\text { Replicate } \\
1\end{array}$ & $\begin{array}{l}\text { Replicate } \\
2\end{array}$ & $\begin{array}{l}\text { Replicate } \\
1\end{array}$ & $\begin{array}{l}\text { Replicate } \\
2\end{array}$ & $\begin{array}{l}\text { Replicate } \\
1\end{array}$ & $\begin{array}{l}\text { Replicate } \\
2\end{array}$ & $\begin{array}{l}\text { Replicate } \\
1\end{array}$ & $\begin{array}{l}\text { Replicate } \\
2\end{array}$ & $\begin{array}{l}\text { Replicate } \\
1\end{array}$ & \begin{tabular}{|l} 
Replicate \\
2
\end{tabular} \\
\hline Hcp & 0.87 & 0.94 & 1.84 & 1.53 & 12.06 & 14.54 & 29.19 & 24.07 & 19.45 & 19.74 \\
\hline TssK & 0.97 & 0.92 & 1.46 & 0.97 & 13.45 & 4.48 & 9.64 & 3.19 & 5.13 & 5.65 \\
\hline TssG & 0.46 & 0.65 & 0.78 & 0.71 & 21.91 & 11.36 & 9.03 & 4.79 & 6.08 & 3.93 \\
\hline TssF & 0.71 & 0.86 & 1.32 & 0.66 & 5.04 & 4.52 & 4.95 & 2.88 & 3.44 & 3.08 \\
\hline TssE & 0.86 & 0.82 & 0.62 & 0.76 & 5.08 & 2.94 & 4.69 & 4.79 & 2.04 & 6.78 \\
\hline VgrG & 0.85 & 1.23 & 1.33 & 0.97 & 3.35 & 2.53 & 4.10 & 3.39 & 3.15 & 4.82 \\
\hline
\end{tabular}

